Article

\title{
Application of Farmyard Manure Rather Than Manure Slurry Mitigates the Net Greenhouse Gas Emissions from Herbage Production System in Nasu, Japan
}

\begin{abstract}
Akinori Mori
Grassland Function Unit, Division of Grassland Farming, Institute of Livestock and Grassland Science, NARO, 768 Senbonmatsu, Nasushiobara, Tochigi 329-2793, Japan; amori@affrc.go.jp; Tel.: +81-287-37-7558

Received: 1 May 2018; Accepted: 9 July 2018; Published: 12 July 2018

Abstract: In Japan, it is important to recycle the nutrients in manure for forage production because most dairy cattle are fed inside, mainly with imported grain and home-grown roughage. To understand the overall effect of manure use on grassland on the net greenhouse gas (GHG) emission and GHG intensity of herbage production systems, the integrated evaluation of emissions of carbon dioxide $\left(\mathrm{CO}_{2}\right)$, methane $\left(\mathrm{CH}_{4}\right)$, and nitrous oxide $\left(\mathrm{N}_{2} \mathrm{O}\right)$ is essential. The objective of this study was to compare the net GHG emissions (expressed in $\mathrm{CO}_{2}$-eq ha ${ }^{-1} \mathrm{y}^{-1}$ ) and GHG intensity (expressed in $\mathrm{CO}_{2}$-eq $\mathrm{Mg}^{-1}$ dry matter yield) of herbage production based on manure slurry + synthetic fertilizer (slurry system) with that based on farmyard manure + synthetic fertilizer (FYM system). Calculations of net GHG emissions and GHG intensity took into account the net ecosystem carbon balance (NECB) in grassland, the $\mathrm{CH}_{4}$ and $\mathrm{N}_{2} \mathrm{O}$ emissions from grassland, and GHG emissions related to cattle waste management, synthetic fertilizer manufacture, and fuel consumption for grassland management based on literature data from previous studies. The net GHG emissions and GHG intensity were 36\% (6.9 $\mathrm{Mg} \mathrm{CO}_{2}$-eq ha $\left.{ }^{-1} \mathrm{y}^{-1}\right)$ and $41 \%\left(0.89 \mathrm{Mg} \mathrm{CO}_{2}\right.$-eq $\left.\mathrm{Mg}^{-1}\right)$, respectively, lower in the FYM system.
\end{abstract}

Keywords: carbon dioxide; cattle waste management; fuel consumption for grassland management; greenhouse gas intensity; lifecycle assessment; methane; net ecosystem carbon balance; nitrous oxide; synthetic fertilizer manufacture

\section{Introduction}

Recent greenhouse gas (GHG) profiles of the agriculture sector in Japan show that major GHG sources in terms of carbon dioxide equivalents $\left(\mathrm{CO}_{2}\right.$-eq) are rice cultivation (methane $\left.\left(\mathrm{CH}_{4}\right), 41 \%\right)$, enteric fermentation $\left(\mathrm{CH}_{4}, 22 \%\right)$, manure management $\left(\mathrm{CH}_{4}\right.$ and nitrous oxide $\left.\left(\mathrm{N}_{2} \mathrm{O}\right), 19 \%\right)$, and soils $\left(\mathrm{N}_{2} \mathrm{O}, 16 \%\right)$ [1]. In Japan, most dairy cattle are fed inside, mainly with imported grain and home-grown roughage. Around $70 \%$ of the dairy cattle waste is composted [2] for use in crop and forage production. Therefore, it is important to apply manure to meadows and pastures, which account for $13.4 \%$ of the total agricultural land area of Japan [3]. In the year 2015, 570,475 ${\mathrm{Mg}-\mathrm{N} \mathrm{y}^{-1}}$ was excreted by livestock in Japan, of which $304,285 \mathrm{Mg}-\mathrm{N} \mathrm{y}^{-1}$ was applied to agricultural soil $(4,496,000 \mathrm{ha})$ [1].

The application of farmyard manure (FYM) to grassland increases the net ecosystem carbon balance (NECB) relative to manure slurry application [4]. This is mainly because the amount of $\mathrm{C}$ input to grassland from FYM is greater than that from slurry, but the decomposition of FYM is slower than that of slurry [4]. Consequently, FYM application has a greater potential to improve the C stock in grassland soil than slurry application. However, emissions of $\mathrm{CH}_{4}$ and $\mathrm{N}_{2} \mathrm{O}$ from manured grassland [5] need to be considered in evaluating the overall effect of manure application on the net GHG emissions from grassland [3]. The soil of grassland usually acts as a sink of atmospheric $\mathrm{CH}_{4}$, 
and manure application only temporarily increases $\mathrm{CH}_{4}$ emission from grassland [3]. In contrast, $\mathrm{N}_{2} \mathrm{O}$ emission increases with increasing the $\mathrm{N}$ surplus in grassland soil [6]. Therefore, judicial application of organic and inorganic $\mathrm{N}$ is necessary to mitigate the $\mathrm{N}_{2} \mathrm{O}$ emission from grassland [3]. Cattle waste management, including slurry storage [7] and composting [8], is another source of GHG, and in addition to $\mathrm{CH}_{4}$ and $\mathrm{N}_{2} \mathrm{O}$ emissions from cattle waste management, farm machinery used for composting FYM also emits $\mathrm{CO}_{2}$ [9]. To maintain productive sward, supplemental fertilizers are used to make up for nutrient insufficiencies in manure (e.g., $\mathrm{N}$ and $\mathrm{P}$ in the case of cattle manure) [5], but their manufacture also emits GHG [10]. Furthermore, GHG emissions from fuel consumption for grassland management also need to be taken into consideration [11].

To assess the net GHG emissions (i.e., integrated evaluation of $\mathrm{CO}_{2}, \mathrm{CH}_{4}$ and $\mathrm{N}_{2} \mathrm{O}$ ) and GHG intensity (GHGI) of herbage production systems, an integrated evaluation of the above processes is necessary (Table S1). On that basis, the identification of important processes with significant contributions is necessary in order to determine the priority of countermeasures to mitigate GHG. To date, it is recognized that the quality and quantity of organic materials applied have great influence on soil organic carbon [4,12]; however, insufficient information is available on the effect of manure type (i.e., slurry or FYM) on the net GHG emissions and GHGI of herbage production systems.

The objectives of this study were: (1) to investigate the net GHG emissions (expressed in $\mathrm{CO}_{2}$-eq ha ${ }^{-1} \mathrm{y}^{-1}$ ) and GHGI (expressed in $\mathrm{CO}_{2}$-eq $\mathrm{Mg}^{-1}$ dry matter yield) of herbage production systems based on manure slurry + synthetic fertilizer (slurry system) and on FYM + synthetic fertilizer (FYM system); (2) to show the relative contributions of each process in GHG emission; and (3) to show how farming practices can be adjusted to minimize emissions. My hypotheses were that the FYM system reduces the net GHG emissions in comparison with the slurry system, and that the contributions of grassland soil and cattle waste management to the net GHG emissions of herbage production systems are greater than the other processes.

\section{Materials and Methods}

\subsection{System Boundary and Functional Units}

The system boundary comprised the following processes: the NECB in grassland, emissions of $\mathrm{CH}_{4}$ and $\mathrm{N}_{2} \mathrm{O}$ from grassland, and GHG emissions related to cattle waste management (i.e., slurry storage and composting FYM), synthetic fertilizer manufacture, and fuel consumption for grassland management operations. The functional unit was defined as ha ${ }^{-1} \mathrm{y}^{-1}$ of grassland or $\mathrm{Mg}^{-1}$ of dry matter yield. The study did not take into account the GHG emissions related to the manufacture of farm machinery and buildings, transport of synthetic fertilizers, or indirect $\mathrm{N}_{2} \mathrm{O}$ emissions related to leaching of nitrate $\left(\mathrm{NO}_{3}{ }^{-}\right)$and redeposition of volatilized ammonia $\left(\mathrm{NH}_{3}\right)$.

\section{2. $\mathrm{NECB}$ and Emissions of $\mathrm{CH}_{4}$ and $\mathrm{N}_{2} \mathrm{O}$ from Grassland}

The NECB and emissions of $\mathrm{CH}_{4}$, and $\mathrm{N}_{2} \mathrm{O}$ from grassland ( 1 ha) treated with slurry (65.8 to $66.4 \mathrm{Mg} \mathrm{ha}^{-1} \mathrm{y}^{-1}$ ) or FYM (36.5 to $39.2 \mathrm{Mg} \mathrm{ha}^{-1} \mathrm{y}^{-1}$ ) were based on previous studies $[4,5]$ in which slurry or FYM was applied to the upper limit based on $\mathrm{K}$ requirement for herbage production. Annualized values of $\mathrm{NECB}$, emissions of $\mathrm{CH}_{4}$, and $\mathrm{N}_{2} \mathrm{O}$ were calculated by averaging the information of two years.

\subsection{GHG Emissions Related to Cattle Waste Management}

Emissions of $\mathrm{CH}_{4}$ and $\mathrm{N}_{2} \mathrm{O}$ from stored slurry were calculated from emission factors (EFs, $3.90 \%$ and $0.02 \%$, respectively) in Japan [1]. Emissions of $\mathrm{CH}_{4}$ and $\mathrm{N}_{2} \mathrm{O}$ from composting of applied FYM were estimated from a farm study [13] and EFs (3.8\% and $2.38 \%$ to $2.39 \%$, respectively) in Japan [1]. Biogenic $\mathrm{CO}_{2}$ losses from manure were excluded (i.e., $\mathrm{C}$ neutral), but emissions of $\mathrm{CO}_{2}$ due to the consumption of electricity or fuel for composting FYM were estimated from a farm study [13] and EFs [14]. Emissions of $\mathrm{CH}_{4}, \mathrm{~N}_{2} \mathrm{O}$ and $\mathrm{CO}_{2}$ per unit area of grassland (1 ha) were calculated by 
multiplying these emissions per unit weight of slurry or FYM and the weight of slurry or FYM annually applied to grassland $\left(\mathrm{Mg} \mathrm{ha}^{-1} \mathrm{y}^{-1}\right)$.

\subsection{GHG Emissions Related to Synthetic Fertilizer Manufacture}

GHG emissions from the manufacture of $\mathrm{N}$ and $\mathrm{P}$ fertilizers were estimated from the SimaPro 7.1 database (PRé Consultants, Amersfoort, Netherlands). Emissions of $\mathrm{CH}_{4}, \mathrm{~N}_{2} \mathrm{O}$ and $\mathrm{CO}_{2}$ per unit area of grassland (1 ha) were calculated by multiplying these emissions per unit weight of synthetic fertilizer and the weight of synthetic fertilizer annually applied to grassland $\left(\mathrm{kg} \mathrm{ha}^{-1} \mathrm{y}^{-1}\right)$. No K fertilizer was used, because the applied slurry or FYM covered the K requirement for herbage production [4,5].

\subsection{GHG Emissions Related to Grassland Management}

GHG emissions due to fuel consumption by farm machinery for loading and spreading of manure and fertilizers and for cutting and harvesting of herbage were estimated from a previous Japanese study in the 1990s [15] and EF [14]. The emission of $\mathrm{CO}_{2}$ per unit area of grassland (1 ha) was calculated by multiplying the fuel consumption per unit of operation, the operation unit necessary for management of grassland (1 ha), and EF.

\subsection{Overall Net GHG Emissions and GHGI of Herbage Production}

Emissions of $\mathrm{CH}_{4}$ and $\mathrm{N}_{2} \mathrm{O}$ were converted to $\mathrm{CO}_{2}$-eq by using values of the 100-year global warming potential, assumed to be 1 for $\mathrm{CO}_{2}, 25$ for $\mathrm{CH}_{4}$, and 298 for $\mathrm{N}_{2} \mathrm{O}$ [16]. The net GHG emissions $\left(\mathrm{CO}_{2}\right.$-eq ha $\left.{ }^{-1} \mathrm{y}^{-1}\right)$ were calculated by considering the NECB and emissions of $\mathrm{CH}_{4}$ and $\mathrm{N}_{2} \mathrm{O}$ from grassland (Section 2.2.) and the GHG emissions related to cattle waste management (Section 2.3.), synthetic fertilizer manufacture (Section 2.4.), and grassland management (Section 2.5.) on an area basis. The GHGI $\left(\mathrm{CO}_{2}\right.$-eq $\left.\mathrm{Mg}^{-1}\right)$ was calculated by dividing the net GHG emissions by the dry matter yield of grassland receiving slurry or FYM [4].

\section{Results and Discussion}

\subsection{NECB and Emissions of $\mathrm{N}_{2} \mathrm{O}$ and $\mathrm{CH}_{4}$ from Grassland}

The NECB of the slurry system $\left(-12.8 \mathrm{Mg} \mathrm{CO}_{2}\right.$-eq ha $\left.{ }^{-1} \mathrm{y}^{-1}\right)$ was far lower than that of the FYM system $\left(-1.8 \mathrm{Mg} \mathrm{CO}_{2}\right.$-eq ha $\left.{ }^{-1} \mathrm{y}^{-1}\right)$; that is, the FYM system contributed more to improving the $\mathrm{C}$ stock in grassland than the slurry system (Figure 1). (Please note that negative NECB values represent net $\mathrm{CO}_{2}$ emission from grassland to the atmosphere.) The NECB of the slurry and FYM systems were similar to previously measured values in Japanese grasslands that respectively received only synthetic fertilizers or FYM + synthetic fertilizers [17], suggesting that slurry C had limited capacity for maintaining soil organic $\mathrm{C}$ in comparison with FYM C [12]. The emissions of $\mathrm{N}_{2} \mathrm{O}$ from grassland were not significantly different between slurry and FYM systems $\left(2.2 \mathrm{Mg} \mathrm{CO}_{2}\right.$-eq ha ${ }^{-1} \mathrm{y}^{-1}$ in slurry system, $2.3 \mathrm{Mg} \mathrm{CO}_{2}$-eq ha ${ }^{-1} \mathrm{y}^{-1}$ in FYM system), because synthetic $\mathrm{N}$ fertilizer was also applied $[18,19]$.

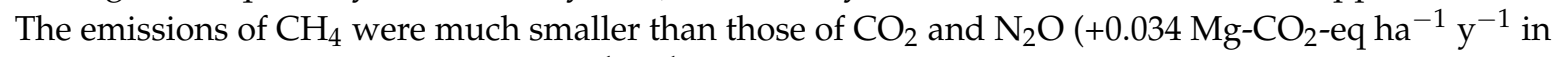
slurry system, $-0.032{\mathrm{Mg}-\mathrm{CO}_{2} \text {-eq ha }}^{-1} \mathrm{y}^{-1}$ in FYM system). 


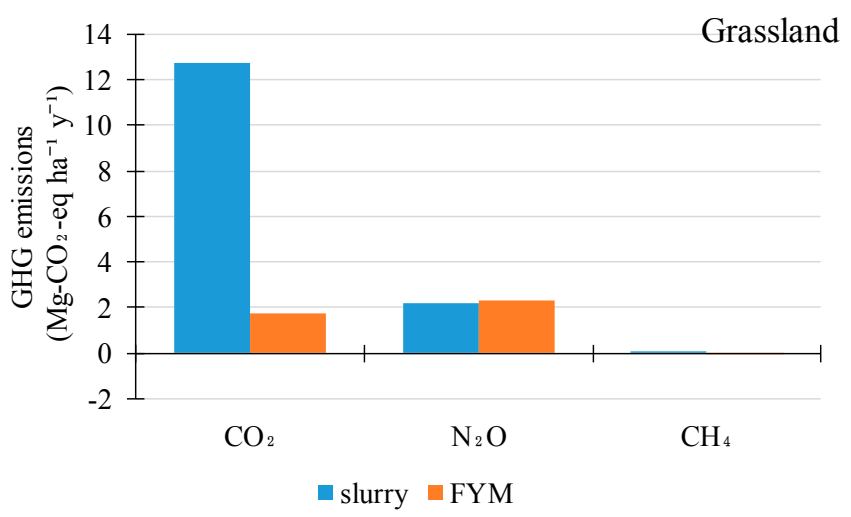

Figure 1. Annualized emissions of $\mathrm{CO}_{2}$ (NECB), $\mathrm{N}_{2} \mathrm{O}$ and $\mathrm{CH}_{4}$ from grassland receiving manure + fertilizer.

\subsection{GHG Emissions Related to Cattle Waste Management}

The GHG emissions related to cattle waste management were due mainly to $\mathrm{CH}_{4}\left(2.9 \mathrm{Mg} \mathrm{CO}_{2}\right.$-eq $\mathrm{ha}^{-1} \mathrm{y}^{-1}$, Table 1$)$ in the slurry system and to both $\mathrm{CH}_{4}\left(4.2 \mathrm{Mg} \mathrm{CO}_{2}\right.$-eq ha ${ }^{-1} \mathrm{y}^{-1}$, Table 2) and $\mathrm{N}_{2} \mathrm{O}$ (2.0 $\mathrm{Mg} \mathrm{CO}{ }_{2}$-eq ha ${ }^{-1} \mathrm{y}^{-1}$, Table 3) in the FYM system (Figure 2). The emission of $\mathrm{CO}_{2}$ related to energy consumption ( $0.15 \mathrm{Mg} \mathrm{CO}_{2}$-eq ha ${ }^{-1} \mathrm{y}^{-1}$, Table 4 ) was smaller than the emission of $\mathrm{CH}_{4}$ and $\mathrm{N}_{2} \mathrm{O}$ by composting. This is because the amount of $\mathrm{CO}_{2}$ emission for composting $1 \mathrm{Mg}$ of FYM was only $4.1 \mathrm{~kg}$. The emission of $\mathrm{N}_{2} \mathrm{O}$ by slurry storage $\left(0.028 \mathrm{Mg}^{-} \mathrm{CO}_{2}\right.$-eq ha ${ }^{-1} \mathrm{y}^{-1}$, Table 5) was much smaller than the emission of $\mathrm{N}_{2} \mathrm{O}$ by composting FYM (2.0 $\mathrm{Mg} \mathrm{CO}_{2}$-eq ha ${ }^{-1} \mathrm{y}^{-1}$, Table 3), mainly due to the small EF for slurry storage $(0.02 \%)$ in comparison with composting FYM $(2.4 \%)$.

Table 1. Emission of $\mathrm{CH}_{4}$ related to storage of dairy cattle slurry.

\begin{tabular}{|c|c|c|c|c|c|c|c|c|c|c|c|c|}
\hline \multicolumn{4}{|c|}{ OM in Slurry $^{1}$} & \multicolumn{4}{|c|}{$\mathrm{CH}_{4}$ Emission $^{2}$} & \multicolumn{5}{|c|}{$\mathrm{CH}_{4}$ Emission $^{3}$} \\
\hline \multicolumn{4}{|c|}{$\operatorname{Mg~ha}^{-1} y^{-1}$} & \multicolumn{4}{|c|}{$\mathrm{kg}-\mathrm{CH}_{4} \mathrm{ha}^{-1} \mathrm{y}^{-1}$} & \multicolumn{5}{|c|}{$\mathrm{Mg}-\mathrm{CO}_{2}$-eq ha ${ }^{-1} \mathrm{y}^{-1}$} \\
\hline \multicolumn{2}{|c|}{ 1st year } & \multicolumn{2}{|c|}{ 2nd year } & \multicolumn{2}{|c|}{ 1st year } & \multicolumn{2}{|c|}{ 2nd year } & \multicolumn{2}{|c|}{ 1st year } & \multicolumn{2}{|c|}{ 2nd year } & \multirow[b]{2}{*}{ Mean } \\
\hline March & May & March & May & March & May & March & May & March & May & March & May & \\
\hline 2.2 & 2.5 & 2.6 & 2.4 & 52 & 60 & 62 & 57 & 1.3 & 1.5 & 1.5 & 1.4 & \\
\hline \multicolumn{2}{|c|}{4.7} & \multicolumn{2}{|c|}{5.0} & \multicolumn{2}{|c|}{113} & \multicolumn{2}{|c|}{119} & \multicolumn{2}{|c|}{2.8} & \multicolumn{2}{|c|}{3.0} & .9 \\
\hline
\end{tabular}

${ }^{1}$ Organic matter content in slurry was determined as $1.764 \times \mathrm{C}$ content. ${ }^{2}$ Emission factor of $\mathrm{CH}_{4}$ from slurry storage was $2.38-2.39 \%\left(\mathrm{~g}-\mathrm{CH}_{4} \mathrm{~g}-\mathrm{OM}^{-1}\right)$ [1]. ${ }^{3}$ The 100-year global warming potential of $\mathrm{CH}_{4}$ was assumed to be 25 [16].

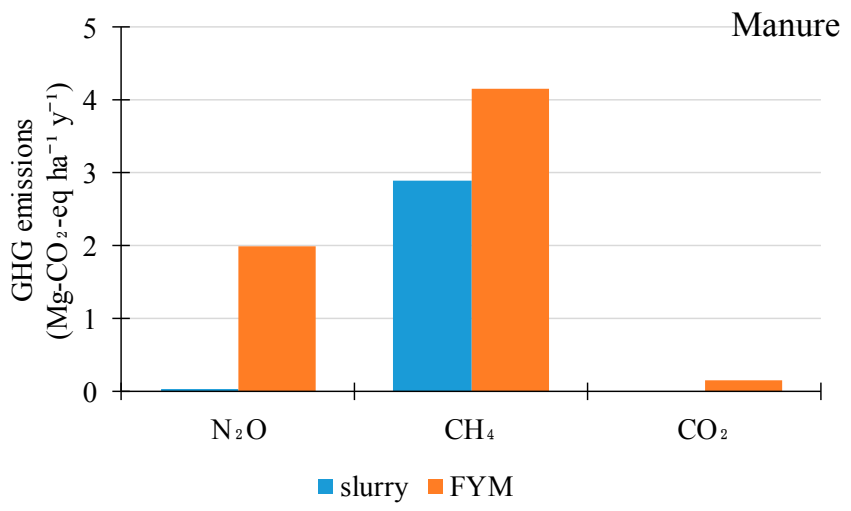

Figure 2. Annualized emissions of $\mathrm{N}_{2} \mathrm{O}, \mathrm{CH}_{4}$, and $\mathrm{CO}_{2}$ related cattle waste management. 
Table 2. Emission of $\mathrm{CH}_{4}$ related to composting.

\begin{tabular}{|c|c|c|c|c|c|c|c|c|c|c|}
\hline \multirow{3}{*}{ Cattle Type } & \multirow{3}{*}{$\begin{array}{c}\text { Number }^{1} \\
\text { Head }\end{array}$} & \multirow{2}{*}{\multicolumn{2}{|c|}{$\begin{array}{c}\text { Excreta }^{2} \\
\text { kg head }^{-1} \mathrm{~d}^{-1}\end{array}$}} & \multirow{3}{*}{$\begin{array}{c}\begin{array}{c}\text { OM in Excreta on } \\
\text { the Farm }\end{array}{ }^{3} \\
\mathrm{~kg}(30 \mathrm{~d})^{-1} \\
\end{array}$} & \multirow{3}{*}{$\begin{array}{c}\begin{array}{c}\mathrm{CH}_{4} \text { Emission on } \\
\text { the Farm }\end{array} \\
\mathrm{kg}^{4}-\mathrm{CH}_{4}(30 \mathrm{~d})^{-1} \\
\end{array}$} & \multirow{2}{*}{\multicolumn{2}{|c|}{$\mathrm{CH}_{4}$ Emission per Unit Weight of FYM ${ }^{5,6}$}} & \multirow{2}{*}{\multicolumn{3}{|c|}{$\begin{array}{c}\mathrm{CH}_{4} \text { Emission per Unit Area of Grassland } \\
{\mathrm{Mg}-\mathrm{CO}_{2} \text {-eq ha }}^{-1} \mathrm{y}^{-1}\end{array}$}} \\
\hline & & & & & & & & & & \\
\hline & & Feces & Urine & & & $\mathrm{kg}-\mathrm{CH}_{4} \mathrm{Mg}^{-1}$ & kg- $\mathrm{CO}_{2}$-eq $\mathrm{Mg}^{-1}$ & 1st year & 2nd year & Mean \\
\hline Lactating & 84.1 & 43 & 14 & 17535 & 666 & \multirow{2}{*}{4.4} & \multirow{2}{*}{110} & \multirow{2}{*}{4.0} & \multirow{2}{*}{4.3} & \multirow{2}{*}{4.2} \\
\hline Non-lactating & 19.9 & 21 & 6 & 2024 & 77 & & & & & \\
\hline
\end{tabular}

Table 3. Emission of $\mathrm{N}_{2} \mathrm{O}$ related to composting dairy FYM.

\begin{tabular}{|c|c|c|c|c|c|c|c|c|c|c|}
\hline \multirow{2}{*}{ Cattle Type } & \multirow{2}{*}{$\begin{array}{c}\text { Number }^{1} \\
\text { Head }\end{array}$} & \multicolumn{2}{|c|}{$\frac{\text { Excreta }^{2}}{\text { kg head }^{-1} \mathrm{~d}^{-1}}$} & \multirow{2}{*}{$\begin{array}{c}\begin{array}{c}\text { in Excreta on the } \\
\text { Farm }^{3}\end{array} \\
\mathrm{~kg}(30 \mathrm{~d})^{-1} \\
\end{array}$} & \multirow{2}{*}{$\begin{array}{l}\begin{array}{l}\mathrm{N}_{2} \mathrm{O} \text { Emission on } \\
\text { the Farm }\end{array}{ }^{4} \\
\mathrm{~kg}_{-}-\mathrm{N}_{2} \mathrm{O}-\mathrm{N}(30 \mathrm{~d})^{-1}\end{array}$} & \multicolumn{2}{|c|}{$\mathrm{N}_{2} \mathrm{O}$ Emission per Unit Weight of FYM $\mathrm{Fr}^{5,6}$} & \multicolumn{3}{|c|}{$\begin{array}{c}\mathrm{N}_{2} \mathrm{O} \text { Emission per Unit Area of Grassland } \\
\mathrm{Mg}^{-\mathrm{CO}_{2}-\mathrm{eq} \mathrm{ha}}{ }^{-1} \mathrm{y}^{-1}\end{array}$} \\
\hline & & Feces & Urine & & & kg- $\mathrm{N}_{2} \mathrm{O} \mathrm{Mg}^{-1}$ & kg-CO ${ }_{2}$-eq $\mathrm{Mg}^{-1}$ & 1st year & 2nd year & Mean \\
\hline Lactating & 84.1 & 43 & 14 & 717 & 17.2 & \multirow[b]{2}{*}{0.18} & \multirow[b]{2}{*}{53} & \multirow[b]{2}{*}{1.9} & \multirow[b]{2}{*}{2.1} & \multirow[b]{2}{*}{2.0} \\
\hline Non-lactating & 19.9 & 21 & 6 & 79 & 1.9 & & & & & \\
\hline
\end{tabular}


Table 4. Emission of $\mathrm{CO}_{2}$ related to energy consumption for composting dairy FYM.

\begin{tabular}{|c|c|c|c|c|c|c|c|}
\hline \multicolumn{2}{|c|}{ Energy Consumption } & \multirow[t]{2}{*}{$\begin{array}{l}\mathrm{CO}_{2} \text { Emission } \\
\text { on the Farm }{ }^{2,3}\end{array}$} & \multirow[t]{2}{*}{$\begin{array}{l}\text { FYM Production } \\
\text { on the Farm }{ }^{1}\end{array}$} & \multirow[t]{2}{*}{$\begin{array}{c}\mathrm{CO}_{2} \text { Emission per } \\
\text { Unit Weight of FYM }\end{array}$} & \multirow{2}{*}{\multicolumn{3}{|c|}{$\begin{array}{c}\mathrm{CO}_{2} \text { Emission per Unit Area of } \\
\text { Grassland }^{4} \\
\mathrm{Mg}-\mathrm{CO}_{2} \mathrm{ha}^{-1} \mathrm{y}^{-1}\end{array}$}} \\
\hline Electricity ${ }^{1}$ & Light Diesel Oil $^{1}$ & & & & & & \\
\hline $\mathrm{kWh}(30 \mathrm{~d})^{-1}$ & $\mathrm{~L}(30 \mathrm{~d})^{-1}$ & $\mathrm{~kg}-\mathrm{CO}_{2}(30 \mathrm{~d})^{-1}$ & $\operatorname{Mg}(30 \mathrm{~d})^{-1}$ & $\mathrm{~kg}-\mathrm{CO}_{2} \mathrm{Mg}^{-1}$ & 1st year & 2nd year & Mean \\
\hline 712.4 & 160 & 688 & 168.8 & 4.1 & 0.15 & 0.16 & 0.15 \\
\hline
\end{tabular}

${ }^{1}$ Based on previous research on the dairy farm from which FYM was collected in this study [13]. ${ }^{2} 378$ g-CO was assumed to be emitted by $1 \mathrm{kWh}$ of electricity consumption [14]. ${ }^{3} 2619 \mathrm{~g}-\mathrm{CO}_{2}$ was assumed to be emitted by consumption of $1 \mathrm{~L}$ light diesel oil [14]. ${ }^{4} \mathrm{CO}_{2}$ emission per unit area of grassland was calculated by multiplying the $\mathrm{CO}_{2}$ emission per unit weight of FYM and the weight of FYM applied to 1 ha of grassland.

Table 5. Emission of $\mathrm{N}_{2} \mathrm{O}$ related to storage of cattle slurry.

\begin{tabular}{|c|c|c|c|c|c|c|c|c|c|c|c|c|}
\hline \multicolumn{4}{|c|}{$\mathrm{N}$ in Slurry } & \multicolumn{4}{|c|}{$\mathrm{N}_{2} \mathrm{O}$ Emission ${ }^{1}$} & \multicolumn{5}{|c|}{$\mathrm{N}_{2} \mathrm{O}$ Emission $^{2}$} \\
\hline \multicolumn{4}{|c|}{ kg-N ha ${ }^{-1} y^{-1}$} & \multicolumn{4}{|c|}{ kg-N ${ }_{2} \mathrm{O}-\mathrm{N} \mathrm{ha} \mathrm{a}^{-1} \mathrm{y}^{-1}$} & \multicolumn{5}{|c|}{$\mathrm{Mg}-\mathrm{CO}_{2}$-eq ha ${ }^{-1} y^{-1}$} \\
\hline \multicolumn{2}{|c|}{ 1st year } & \multicolumn{2}{|c|}{ 2nd year } & \multicolumn{2}{|c|}{ 1st year } & \multicolumn{2}{|c|}{ 2nd year } & \multicolumn{2}{|c|}{ 1st year } & \multicolumn{2}{|c|}{ 2nd year } & \multirow{2}{*}{ Mean } \\
\hline March & May & March & May & March & May & March & May & March & May & March & May & \\
\hline 150 & 150 & 150 & 150 & 0.030 & 0.030 & 0.030 & 0.030 & 0.014 & 0.014 & 0.014 & 0.014 & \\
\hline \multicolumn{2}{|c|}{300} & \multicolumn{2}{|c|}{300} & \multicolumn{2}{|c|}{0.060} & \multicolumn{2}{|c|}{0.060} & \multicolumn{2}{|c|}{0.028} & \multicolumn{2}{|c|}{0.028} & 0.028 \\
\hline
\end{tabular}

${ }^{1}$ Emission factor of $\mathrm{N}_{2} \mathrm{O}$ from slurry storage was $0.02 \%\left(\mathrm{~g}-\mathrm{N}_{2} \mathrm{O}-\mathrm{N}\right.$ g-N $\left.{ }^{-1}\right)$ [1]. ${ }^{2}$ The 100-year global warming potential of $\mathrm{N}_{2} \mathrm{O}$ was assumed to be 298 [16].

\subsection{GHG Emissions Related to Fertilizer Manufacture}

The GHG emission related to fertilizer manufacture in the FYM system $\left(1.5 \mathrm{Mg} \mathrm{CO}_{2}\right.$-eq ha ${ }^{-1} \mathrm{y}^{-1}$, Table 6) was almost double that for the slurry system (0.82 $\mathrm{Mg} \mathrm{CO}_{2}$-eq ha ${ }^{-1} \mathrm{y}^{-1}$, Table 6), mainly owing to the difference in $\mathrm{N}$ application rate. Slurry contains substantial amount of readily available $\mathrm{N}$, however, most of $\mathrm{N}$ in FYM is in organic form. Therefore, the amount of $\mathrm{N}$ fertilizer supplemented to grassland in FYM system (159 to $177 \mathrm{~kg}-\mathrm{N} \mathrm{y}^{-1}$ ) was greater than that in slurry system $\left(90 \mathrm{~kg}^{-\mathrm{N} \mathrm{y}^{-1}}\right)$.

\subsection{GHG Emissions Related to Grassland Management}

The GHG emissions related to grassland management were similar between the FYM and slurry systems ( 0.49 vs. $0.47 \mathrm{Mg} \mathrm{CO}_{2}$-eq ha ${ }^{-1} \mathrm{y}^{-1}$, Table 7 ), because the herbage yields were not significantly different between the slurry $\left(8.8 \mathrm{Mg} \mathrm{y}^{-1}\right)$ and FYM $\left(9.5 \mathrm{Mg} \mathrm{y}^{-1}\right)$ systems. The GHG emissions related to grass cutting, turning and harvesting, bailing and wrapping were greater than those related to loading and spreading of slurry or FYM. This is mainly because slurry and FYM were spread twice and once a year, respectively; however, grass cutting, turning and harvesting, bailing and wrapping was performed four times a year in both the slurry and FYM systems. 
Table 6. Emissions of GHG related to synthetic fertilizer manufacture.

\begin{tabular}{|c|c|c|c|c|c|c|c|c|c|c|c|c|}
\hline & \multirow{3}{*}{\multicolumn{2}{|c|}{$\begin{array}{c}\text { Emission from Synthetic N Fertilizer Manufacture } \\
\text { per Unit Weight }{ }^{1,2}\end{array}$}} & \multirow{2}{*}{\multicolumn{4}{|c|}{$\begin{array}{c}\mathrm{N} \text { application Rate } \\
\mathrm{kg}-\mathrm{N} \mathrm{ha}{ }^{-1} \mathrm{y}^{-1}\end{array}$}} & \multirow{2}{*}{\multicolumn{6}{|c|}{$\begin{array}{c}\text { GHG Emissions } \\
{\mathrm{Mg}-\mathrm{CO}_{2}-\mathrm{eq} \mathrm{ha}}^{-1} \mathrm{y}^{-1}\end{array}$}} \\
\hline & & & & & & & & & & & & \\
\hline & & & \multicolumn{2}{|c|}{ 1st year } & \multicolumn{2}{|c|}{ 2nd year } & \multicolumn{2}{|c|}{ 1st year } & \multicolumn{2}{|c|}{ 2nd year } & \multicolumn{2}{|c|}{ Mean } \\
\hline & kg Mg-N ${ }^{-1}$ & kg-CO ${ }_{2}$-eq Mg-N ${ }^{-1}$ & Slurry & FYM & Slurry & FYM & Slurry & FYM & Slurry & FYM & Slurry & FYM \\
\hline $\mathrm{CO}_{2}$ & 2769 & 2769 & & & & & & & & & & \\
\hline $\mathrm{CH}_{4}$ & 0.13 & 3 & 90 & 177 & 90 & 159 & 0.77 & 1.51 & 0.77 & 1.36 & 0.77 & 1.43 \\
\hline \multirow[t]{5}{*}{$\mathrm{N}_{2} \mathrm{O}$} & 19.3 & 5751 & & & & & & & & & & \\
\hline & \multirow{3}{*}{\multicolumn{2}{|c|}{$\begin{array}{c}\text { Emission from Synthetic P Fertilizer Manufacture } \\
\text { per Unit Weight }{ }^{1,2}\end{array}$}} & \multicolumn{4}{|c|}{$\mathrm{P}_{2} \mathrm{O}_{5}$ Application Rate } & \multicolumn{6}{|c|}{ GHG Emissions } \\
\hline & & & \multicolumn{4}{|c|}{ kg- $\mathrm{P}_{2} \mathrm{O}_{5} \mathrm{ha}^{-1} \mathrm{y}^{-1}$} & \multicolumn{6}{|c|}{ kg-CO ${ }_{2}$-eq ha ${ }^{-1} y^{-1}$} \\
\hline & & & \multicolumn{2}{|c|}{ 1st year } & \multicolumn{2}{|c|}{ 2nd year } & \multicolumn{2}{|c|}{ 1st year } & \multicolumn{2}{|c|}{ 2nd year } & \multicolumn{2}{|c|}{ Mean } \\
\hline & $\mathrm{kg} \mathrm{Mg}-\mathrm{P}_{2} \mathrm{O}_{5}{ }^{-1}$ & kg-CO ${ }_{2}$-eq $\mathrm{Mg}-\mathrm{P}_{2} \mathrm{O}_{5}-1$ & Slurry & FYM & Slurry & FYM & Slurry & FYM & Slurry & FYM & Slurry & FYM \\
\hline $\mathrm{CO}_{2}$ & 1117 & 1117 & & & & & & & & & & \\
\hline $\mathrm{CH}_{4}$ & 2.07 & 52 & 49 & 67 & 46 & 45 & 0.06 & 0.08 & 0.05 & 0.05 & 0.06 & 0.07 \\
\hline \multirow[t]{5}{*}{$\mathrm{N}_{2} \mathrm{O}$} & 0.038 & 11 & & & & & & & & & & \\
\hline & \multirow{3}{*}{\multicolumn{2}{|c|}{$\begin{array}{c}\text { Emission from Synthetic K Fertilizer Manufacture } \\
\text { per Unit Weight }{ }^{1,2}\end{array}$}} & \multicolumn{4}{|c|}{$\mathrm{K}_{2} \mathrm{O}$ Application Rate } & \multicolumn{6}{|c|}{ GHG Emissions } \\
\hline & & & \multicolumn{4}{|c|}{$\mathrm{kg}-\mathrm{K}_{2} \mathrm{O} \mathrm{ha}^{-1} \mathrm{y}^{-1}$} & \multicolumn{6}{|c|}{ kg-CO ${ }_{2}$-eq ha ${ }^{-1} y^{-1}$} \\
\hline & & & \multicolumn{2}{|c|}{ 1st year } & \multicolumn{2}{|c|}{ 2nd year } & \multicolumn{2}{|c|}{ 1st year } & \multicolumn{2}{|c|}{ 2nd year } & \multicolumn{2}{|c|}{ Mean } \\
\hline & $\mathrm{kg} \mathrm{Mg}-\mathrm{K}_{2} \mathrm{O}^{-1}$ & $\mathrm{~kg}-\mathrm{CO}_{2}$-eq $\mathrm{Mg}-\mathrm{K}_{2} \mathrm{O}^{-1}$ & Slurry & FYM & Slurry & FYM & Slurry & FYM & Slurry & FYM & Slurry & FYM \\
\hline $\mathrm{CO}_{2}$ & 617 & 617 & & & & & & & & & & \\
\hline $\mathrm{CH}_{4}$ & 1.38 & 35 & 0 & 0 & 0 & 0 & 0.00 & 0.00 & 0.00 & 0.00 & 0.00 & 0.00 \\
\hline $\mathrm{N}_{2} \mathrm{O}$ & 0.049 & 15 & & & & & & & & & & \\
\hline Total & & & & & & & 0.82 & 1.6 & 0.82 & 1.4 & 0.82 & 1.5 \\
\hline
\end{tabular}

${ }^{1}$ Based on the SimaPro 7.1 database. ${ }^{2}$ The 100-year global warming potential was assumed to be 1 for $\mathrm{CO}_{2}, 25$ for $\mathrm{CH}_{4}$, and 298 for $\mathrm{N}_{2} \mathrm{O}$ [16]. 
Table 7. Emission of $\mathrm{CO}_{2}$ related to fuel consumption for grassland management and transport.

\begin{tabular}{|c|c|c|c|c|c|c|c|c|c|c|c|c|}
\hline \multirow{2}{*}{\multicolumn{2}{|c|}{ Machine Operation }} & \multirow{3}{*}{$\begin{array}{c}\begin{array}{c}\text { Consumption of Light Diesel } \\
\text { Oil per Operating Unit }\end{array} \\
\mathrm{L} \mathrm{ha}^{-1} \text { unit }^{-1} \\
\end{array}$} & \multicolumn{4}{|c|}{ Operating Unit } & \multicolumn{6}{|c|}{ 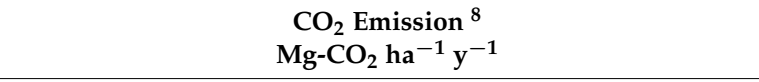 } \\
\hline & & & \multicolumn{2}{|c|}{ 1st year } & \multicolumn{2}{|c|}{ 2nd year } & \multicolumn{2}{|c|}{ 1st year } & \multicolumn{2}{|c|}{ 2nd year } & \multicolumn{2}{|c|}{ Mean } \\
\hline & & & Slurry & FYM & Slurry & FYM & Slurry & FYM & Slurry & FYM & Slurry & FYM \\
\hline Loading FYM $^{1}$ & $30 \mathrm{Mg} \mathrm{unit}^{-1}$ & 10.0 & 0 & 1.22 & 0 & 1.31 & 0.00 & 0.03 & 0.00 & 0.03 & 0.00 & 0.03 \\
\hline FYM transport 1,2 & $30 \mathrm{Mg} \mathrm{unit}^{-1}$ & 2.15 & 0 & 1.22 & 0 & 1.31 & 0.00 & 0.01 & 0.00 & 0.01 & 0.00 & 0.01 \\
\hline FYM spreading ${ }^{1}$ & $30 \mathrm{Mg} \mathrm{unit}^{-1}$ & 3.3 & 0 & 1.22 & 0 & 1.31 & 0.00 & 0.01 & 0.00 & 0.01 & 0.00 & 0.01 \\
\hline Slurry transport 2,3 & $80 \mathrm{Mg} \mathrm{unit}^{-1}$ & 18.4 & 0.83 & 0 & 0.82 & 0 & 0.04 & 0.00 & 0.04 & 0.00 & 0.04 & 0.00 \\
\hline Slurry spreading ${ }^{3}$ & $80 \mathrm{Mg} \mathrm{unit}^{-1}$ & 3.8 & 0.83 & 0 & 0.82 & 0 & 0.01 & 0.00 & 0.01 & 0.00 & 0.01 & 0.00 \\
\hline Fertilizer distribution 2,4 & $500 \mathrm{~kg} \mathrm{unit}^{-1}$ & 2.4 & 1.42 & 2.45 & 1.38 & 2.03 & 0.01 & 0.02 & 0.01 & 0.01 & 0.01 & 0.01 \\
\hline Grass cutting $^{2}$ & ha unit $^{-1}$ & 8.1 & 4 & 4 & 4 & 4 & 0.08 & 0.08 & 0.08 & 0.08 & 0.08 & 0.08 \\
\hline Turning and harvesting ${ }^{2}$ & ha unit ${ }^{-1}$ & 15.35 & 4 & 4 & 4 & 4 & 0.16 & 0.16 & 0.16 & 0.16 & 0.16 & 0.16 \\
\hline Bailing haylage ${ }^{2,5}$ & $7 \mathrm{Mg}-\mathrm{DM}$ unit $^{-1}$ & 19.6 & 1.10 & 1.21 & 1.41 & 1.50 & 0.06 & 0.06 & 0.07 & 0.08 & 0.06 & 0.07 \\
\hline Wrapping haylage 2,6 & $3 \mathrm{Mg}-\mathrm{DM}$ unit $^{-1}$ & 11.1 & 2.57 & 2.83 & 3.30 & 3.50 & 0.07 & 0.08 & 0.10 & 0.10 & 0.09 & 0.09 \\
\hline Haylage transport 2,7 & $7 \mathrm{Mg}-\mathrm{DM}$ unit $^{-1}$ & 5.6 & 1.10 & 1.21 & 1.41 & 1.50 & 0.02 & 0.02 & 0.02 & 0.02 & 0.02 & 0.02 \\
\hline Total & & & & & & & & & & & 0.47 & 0.49 \\
\hline
\end{tabular}

Loading, transport, and spreading of $30 \mathrm{Mg}$-FYM was assumed to be 1 operating unit [15]. ${ }^{2}$ Grassland was assumed to be $500 \mathrm{~m}$ from cowshed [15]. ${ }^{3}$ Loading, transport, and spreading of $80 \mathrm{Mg}$-slurry was assumed to be 1 operating unit [15]. ${ }^{4}$ Distribution of $500 \mathrm{~kg}$ fertilizer was assumed to be 1 operating unit [15]. ${ }^{5}$ Bailing of $7 \mathrm{Mg}$-DM haylage was assumed to be 1 operating unit [15]. ${ }^{6}$ Wrapping of $3 \mathrm{Mg}$-DM haylage was assumed to be 1 operating unit [15]. ${ }^{7}$ Transport of $7 \mathrm{Mg}$-DM haylage was assumed to be 1 operating unit [15]. ${ }^{8} 2619 \mathrm{~g}$-CO 2 was assumed to be emitted by consumption of $1 \mathrm{~L}$ light diesel oil [14] 


\subsection{Overall Net GHG Emissions and GHGI}

The net GHG emissions was $19 \mathrm{Mg} \mathrm{CO}_{2}$-eq ha ${ }^{-1} \mathrm{y}^{-1}$ in the slurry system and $12 \mathrm{Mg} \mathrm{CO}_{2}$-eq $\mathrm{ha}^{-1} \mathrm{y}^{-1}$ in the FYM system (Figure 3). The GHGI was $2.2 \mathrm{Mg} \mathrm{CO}_{2}$-eq $\mathrm{Mg}^{-1}$ in the slurry system and $1.3 \mathrm{Mg} \mathrm{CO}_{2}$-eq $\mathrm{Mg}^{-1}$ in the FYM system. Thus, the net GHG emissions of the FYM system was $36 \%$ (6.9 $\mathrm{Mg} \mathrm{CO}_{2}$-eq ha $\left.{ }^{-1} \mathrm{y}^{-1}\right)$ less and the GHGI of the FYM system was $41 \%\left(0.89 \mathrm{CO}_{2}\right.$-eq $\left.\mathrm{Mg}^{-1}\right)$ less than that of the slurry system.

The contribution of grassland soil, cattle waste management, fertilizer manufacture and grassland management to the net GHG emissions were $78 \%\left(\mathrm{CO}_{2}: 66 \%, \mathrm{~N}_{2} \mathrm{O}: 11 \%\right.$ and $\left.\mathrm{CH}_{4}: 0.2 \%\right)$, $15 \%\left(\mathrm{CO}_{2}: 0.0 \%, \mathrm{CH}_{4}: 15 \%, \mathrm{~N}_{2} \mathrm{O}: 0.1 \%\right), 4 \%$ and $2 \%$ in the slurry system, and $33 \%\left(\mathrm{CO}_{2}: 14 \%, \mathrm{~N}_{2} \mathrm{O}: 19 \%\right.$ and $\left.\mathrm{CH}_{4}:-0.3 \%\right), 51 \%\left(\mathrm{CO}_{2}: 1 \%, \mathrm{~N}_{2} \mathrm{O}: 16 \%, \mathrm{CH}_{4}: 34 \%\right), 12 \%$ and $4 \%$ in the FYM system, respectively. These results collectively suggest that NECB and the $\mathrm{N}_{2} \mathrm{O}$ emissions from grassland and the $\mathrm{CH}_{4}$ and $\mathrm{N}_{2} \mathrm{O}$ emissions related cattle waste management are crucial to the control of net GHG emission and GHGI.

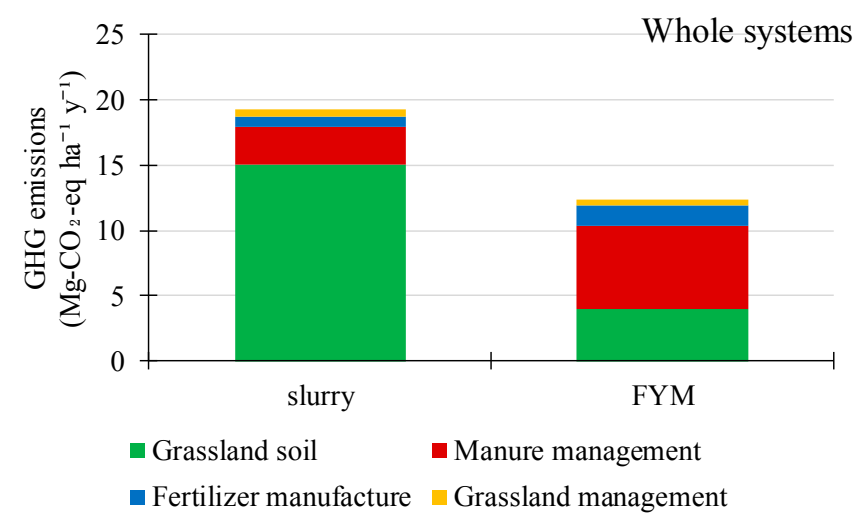

Figure 3. Annualized overall net GHG (greenhouse gas) emissions from herbage production.

The FYM system reduced the net GHG emissions and GHGI relative to the slurry system (Figure 3). The net reduction was due largely to the improvement of $C$ stock in grassland (Figure 1). Although the emissions of GHG related to cattle waste management and fertilizer manufacture were greater in the FYM system than in the slurry system (Figure 3), the FYM system maintained an advantage in net GHG emissions and GHGI, due mainly to the difference in NECB in grassland (Figure 1)-that is, the persistent organic matter in FYM decomposed slowly in the soil and contributed to the improvement of $\mathrm{C}$ stock, but the labile organic matter in slurry decomposed quickly in the soil and was released to the atmosphere as $\mathrm{CO}_{2}$ [4]. Our results support the validity of FYM application for the mitigation of GHG emissions [20,21], not only from grassland, but also during herbage production. In the slurry and FYM systems, $\mathrm{N}$ and $\mathrm{P}$ were supplemented based on the fertilizer recommendation. Therefore, the yields in the slurry $\left(8.8 \mathrm{Mg} \mathrm{y}^{-1}\right)$ and FYM $\left(9.5 \mathrm{Mg} \mathrm{y}^{-1}\right)$ systems were comparable to the standard yield $\left(8-10 \mathrm{Mg}^{-1}\right)$ in Nasu, Japan [4].

\subsection{Adjustment of Farming Practices}

These results show that the FYM system improved the net GHG emissions and GHGI relative to the slurry system (Figure 3). In Japan, a substantial amount of manure is derived from imported feed, and thus represents the net import of organic matter, which must be used with care for fertility management [3]. Making maximum use of manure in consideration of $\mathrm{N}, \mathrm{P}$, and $\mathrm{K}$ requirements for herbage production to reduce synthetic fertilizer rates to the absolute minimum is crucial to mitigating overall GHG emissions [18,22]. Applying manure and synthetic $\mathrm{N}$ fertilizer in excess of demand increases $\mathrm{N}_{2} \mathrm{O}$ emissions from grassland $[23,24]$. Therefore, the tightening of $\mathrm{N}$ application rates can limit overall GHG emissions. Mixing low-quality dried grass as a bulking agent into FYM 
reduced $\mathrm{CH}_{4}$ and $\mathrm{N}_{2} \mathrm{O}$ emissions [2] and could further improve the $\mathrm{C}$ stock in grassland. For this goal, both the selection of appropriate methods for cattle waste management and the decision to base fertilizer application rates on the $\mathrm{N}$ supply from manure are crucial to reducing the net GHG emissions and GHGI.

\section{Conclusions}

The FYM system reduced the overall net GHG emissions and GHGI by $36 \%$ (6.9 $\mathrm{Mg} \mathrm{CO}_{2}$-eq ha ${ }^{-1} \mathrm{y}^{-1}$ ) and $41 \%\left(0.89 \mathrm{Mg} \mathrm{CO}_{2}\right.$-eq $\left.\mathrm{Mg}^{-1}\right)$, respectively, relative to the slurry system. The net reduction was due largely to the improvement of $C$ stock in grassland. Although the emission of GHG related to cattle waste management and supplemental fertilizer manufacture was greater in the FYM system than in the slurry system, the FYM system maintained an advantage. NECB and the $\mathrm{N}_{2} \mathrm{O}$ emissions from grassland and the $\mathrm{CH}_{4}$ and $\mathrm{N}_{2} \mathrm{O}$ emissions related to cattle waste management are crucial to the control of net GHG emissions and GHGI.

Supplementary Materials: The following is available online at http://www.mdpi.com/2073-4433/9/7/261/s1, Table S1: Calculation bases of greenhouse gas emissions.

Acknowledgments: This work was financially supported by the Ministry of Agriculture, Forestry and Fisheries of Japan through the "Development of technologies for mitigation and adaptation to climate change in Agriculture, Forestry and Fisheries" research project. The author thanks Ryusuke Hatano, Hokkaido University, and Kazuyuki Yagi, NARO, for their valuable discussion on the experimental design.

Conflicts of Interest: The author declares no conflict of interest. The funding body had no role in the collection, analyses, or interpretation of data, in the writing of the manuscript, or in the decision to publish the results.

\section{References}

1. Greenhouse Gas Inventory Office of Japan (GIO). National Greenhouse Gas Inventory Report of Japan; Center for Global Environmental Research, National Institute for Environmental Studies: Tsukuba, Japan, 2017.

2. Maeda, K.; Hanajima, D.; Morioka, R.; Toyoda, S.; Yoshida, N.; Osada, T. Mitigation of greenhouse gas emission from the cattle manure composting process by use of a bulking agent. Soil Sci. Plant Nutr. 2013, 59, 96-106. [CrossRef]

3. Mori, A. Greenhouse gas sink-source functions of grassland ecosystems. Jpn. Agric. Res. Q. 2016, 50, 187-190. [CrossRef]

4. Mori, A.; Hojito, M. Effect of dairy manure type on the carbon balance of mowed grassland in Nasu, Japan: Comparison between manure slurry plus synthetic fertilizer plots and farmyard manure plus synthetic fertilizer plots. Soil Sci. Plant Nutr. 2015, 61, 736-746. [CrossRef]

5. Mori, A.; Hojito, M. Effect of dairy manure type and supplemental synthetic fertilizer on methane and nitrous oxide emissions from a grassland in Nasu, Japan. Soil Sci. Plant Nutr. 2015, 61, 347-358. [CrossRef]

6. Shimizu, M.; Marutani, S.; Desyatkin, A.R.; Jin, T.; Nakano, K.; Hata, H.; Hatano, R. Nitrous oxide emissions and nitrogen cycling in managed grassland in Southern Hokkaido, Japan. Soil Sci. Plant Nutr. 2010, 56, 676-688. [CrossRef]

7. Sawamoto, T.; Nakamura, M.; Nekomoto, K.; Hoshiba, S.; Minato, K.; Nakayama, M.; Osada, T. The cumulative methane production from dairy cattle slurry can be explained by its volatile solid, temperature and length of storage. Anim. Sci. J. 2016, 87, 827-834. [CrossRef] [PubMed]

8. Fukumoto, Y.; Suzuki, K.; Waki, M.; Yasuda, T. Mitigation option of greenhouse gas emissions from livestock manure composting. Jpn. Agric. Res. Q. 2015, 49, 307-312. [CrossRef]

9. Zhang, H.; Matsuto, T. Comparison of mass balance, energy consumption and cost of composting facilities for different types of organic waste. Waste Manag. 2011, 31, 416-422. [CrossRef] [PubMed]

10. Snyder, C.S.; Bruulsema, T.W.; Jensen, T.L.; Fixen, P.E. Review of greenhouse gas emissions from crop production systems and fertilizer management effects. Agric. Ecosyst. Environ. 2009, 133, 247-266. [CrossRef]

11. Pimentel, D. Handbook of Energy Utilization in Agriculture; CRC Press: Boca Raton, FL, USA, 1980.

12. Bhogal, A.; Nicholson, F.A.; Rollett, A.; Taylor, M.; Litterick, A.; Whittingham, M.J.; Williams, J.R. Improvements in the quality of agricultural soils following organic material additions depend on both the quantity and quality of the materials applied. Front. Sustain. Food Syst. 2018, 2, 9. [CrossRef] 
13. Ito, N. Open type compost fermentation instrument. In The Report of Cost Research of Machines and Equipment for Livestock Waste Treatments 2002 (Undertake Research Project Commissioned by Livestock Industry's Environmental Improvement Organization); Japan Livestock Production Facilities and Machineries Association: Tokyo, Japan, 2002; pp. 46-73. (In Japanese)

14. National Institute for Agro-Environmental Sciences (NIAES). Manual for Life Cycle Assessment of Agricultural Practice in Japan; National Institute for Agro-Environmental Sciences: Tsukuba, Japan, 2003; pp. 1-51. (In Japanese)

15. Sasaki, Y. Forage Crops, Specific Energy Consumption for Production Systems of Major Crops; Agriculture, Forestry and Fisheries Technical Information Society: Tokyo, Japan, 1996; pp. 361-383. (In Japanese)

16. Intergovernmental Panel on Climate Change (IPCC). Contribution of Working Group I to the Fourth Assessment Report of the Intergovernmental Panel on Climate Change; Climate Change 2007: The Physical Science Basis; Solomon, S., Qin, D., Manning, M., Chen, Z., Marquis, M., Averyt, K.B., Tignor, M., Miller, H.L., Eds.; Cambridge University Press: Cambridge, UK, 2007.

17. Shimizu, M.; Marutani, S.; Desyatkin, A.R.; Jin, T.; Hata, H.; Hatano, R. The effect of manure application on carbon dynamics and budgets in a managed grassland of Southern Hokkaido, Japan. Agric. Ecosyst. Environ. 2009, 130, 31-40. [CrossRef]

18. Handbook of Animal Waste Management and Utilization in Hokkaido 2004; Ohara, M.; Takeda, Y.; Omura, K. (Eds.) Hokkaido Prefectural Experiment Stations and Hokkaido Animal Research Center: Sapporo, Japan, 2004. (In Japanese)

19. Shiga, H.; Ohyama, N.; Maeda, K.; Suzuki, M. An evaluation of different organic materials based on their decomposition pattern in paddy soils. Res. Bull. Natl. Agric. Res. Cetr. 1985, 5, 1-19, (In Japanese with English Summary).

20. Hirata, R.; Miyata, A.; Mano, M.; Shimizu, M.; Arita, T.; Kouda, Y.; Matsuura, S.; Niimi, M.; Mori, A.; Saigusa, T.; et al. Carbon dioxide exchange at four intensively managed grassland sites across different climate zones of Japan and the influence of manure application on ecosystem carbon and greenhouse gas budgets. Agric. For. Meteorol. 2013, 177, 57-68. [CrossRef]

21. Shimizu, M.; Hatano, R.; Arita, T.; Kouda, Y.; Mori, A.; Matsuura, S.; Niimi, M.; Jin, T.; Desyatkin, A.R.; Kawamura, O; et al. The effect of fertilizer and manure application on $\mathrm{CH}_{4}$ and $\mathrm{N}_{2} \mathrm{O}$ emissions from managed grasslands in Japan. Soil Sci. Plant Nutr. 2013, 59, 69-86. [CrossRef]

22. Grassland Agriculture and Forage Seed Association (GAFSA). Agricultural activity and global warming. In Greenhouse Gas Mitigation by Self-Supplying Forage Production; Grassland Agriculture and Forage Seed Association: Tokyo, Japan, 2010; pp. 3-7. (In Japanese)

23. Cardenas, L.M.; Thorman, R.; Ashlee, N.; Butler, M.; Chadwick, D.; Chambers, B.; Cuttle, S.; Donovan, N.; Kingston, H.; Lane, $\mathrm{S}$; ; et al. Quantifying annual $\mathrm{N}_{2} \mathrm{O}$ emission fluxes from grazed grassland under a range of inorganic fertiliser nitrogen inputs. Agric. Ecosyst. Environ. 2010, 136, 218-226. [CrossRef]

24. Paustian, K.; Lehmann, J.; Ogle, S.; Reay, D.; Robertson, G.P.; Smith, P. Climate-smart soils. Nature 2016, 532, 49-57. [CrossRef] [PubMed]

(C) 2018 by the author. Licensee MDPI, Basel, Switzerland. This article is an open access article distributed under the terms and conditions of the Creative Commons Attribution (CC BY) license (http:// creativecommons.org/licenses/by/4.0/). 